\title{
LAS ELECCIONES PARA GOBERNADOR EN SONORA DURANTE LA ALTERNANCIA POLÍTICA (1997-2015)
}

Francisco Alán Collins Castillo ${ }^{1}$

Sumario: I.Introducción. II.Procesos electorales. III.Conclusiones

IV.Referencias

\section{Resumen}

Este artículo aborda los cambios acontecidos en los procesos electorales para elegir al titular del Ejecutivo estatal desde 1997, año en que inició el proceso de pluralización política en el estado, hasta 2015, fecha en que se llevaron a cabo las elecciones más recientes para elegir gobernador. Para esto se utilizó el concepto de "Cartelización" para analizar la manera en que los partidos políticos en general han cambiado su manera de operar durante las elecciones, pasando de estar organizados en torno a una ideología a desecharla, y en su lugar tener como objetivo casi único ganar elecciones; además, se consultaron diversos estudios académicos, así como notas periodísticas referentes al período estudiado. Las conclusiones más relevantes que arrojó la investigación consisten en la consolidación de un sistema bipartidista, y la importancia que han adquirido las campañas sucias, utilizadas de manera predominante entre los candidatos para desprestigiar a sus adversarios políticos.

Palabras clave: Partidos políticos, elecciones, campañas sucias

${ }^{1}$ Licenciado en Sociología y Licenciado en Derecho, Universidad de Sonora. Actualmente es asistente de investigación en El Colegio de Sonora.

Correo electrónico: acollins@colson.edu.mx 


\title{
ELECTIONS FOR GOVERNOR IN SONORA DURING POLITICAL ALTERNANCE (1997-2015)
}

Summary: I. Introduction. II.Electoral processes. III.Conclusions

IV.References

\begin{abstract}
:
This article deals with the changes that have taken place in the electoral processes to elect the head of the State Executive since 1997, the year in which the process of political pluralization began in the state, until 2015, the date on which the most recent elections to elect governor. For this, the concept of "Cartelización" was used to analyze the way in which political parties in general have changed their way of operating during elections, going from being organized around an ideology to discarding it, and instead having as Almost unique goal winning elections; In addition, several academic studies were consulted, as well as journalistic notes referring to the period studied. The most relevant conclusions that the investigation showed are the consolidation of a bipartisan system, and the importance that dirty campaigns have acquired, used predominantly among the candidates to discredit their political opponents.
\end{abstract}

Key words: Political parties, elections, smear campaign

\section{INTRODUCCIÓN}

Como señala Covarrubias ${ }^{2}$ en 1997 comenzó un proceso que llevó a la política regional a regirse por dos elementos: las alianzas y acuerdos a trasmano en los partidos y entre los partidos para conservar el poder y la

${ }^{2}$ Covarrubias, Alejandro. «La alternancia en Sonora. De legacías y divisiones a la cartelización de la política.» En Estudios sobre Sonora 2010, de Eloy Méndez y Alejandro Covarrubias. Hermosillo: Mora-Cantúa Editores, 2011, p. 56 
priorización de las luchas internas en las tomas de decisiones de las dirigencias partidarias, dirigidas a eliminar primero la competencia interna. Dicho autor, para explicar la transformación de la vida política en el estado, propone analizarla desde la teoría de la "cartelización”.

La "cartelización" consiste en el manejo de los partidos políticos como si fueran empresas, en vez de ser manejadas como organismos que buscan el cambio en los asuntos públicos. Esto lleva por consiguiente a buscar minimizar las pérdidas ocasionadas por las derrotas en los comicios, creando redes de apoyo entre los líderes de los partidos y personas ajenas a éste, incluso con personas pertenecientes a otros partidos, buscando más proteger los intereses del partido que proteger el interés de la población en general, cumpliendo así lo dicho por Michels $^{3}$ con respecto a la formación de las oligarquías en los partidos políticos.

Esta cartelización surge en Sonora debido a que, por los cambios acontecidos en la política nacional, como la pérdida de la hegemonía priista en el Poder Legislativo y una cierta indiferencia por parte del presidente Ernesto Zedillo con respecto al PRI, los poderes locales en los estados de la república adquieren mayor protagonismo. Comienzan a depender menos de las decisiones del Ejecutivo Federal, y más de sus propias decisiones para conservar el poder, por lo que las distintas facciones políticas tienen que realizar acuerdos, ya sea en el Poder Legislativo, ya sea en los ayuntamientos locales, lo que contribuye a homogeneizar a los partidos políticos y a sus representantes, pasando a un segundo plano la ideología de los mismos. Esto lleva a que, durante los procesos electorales, tanto candidatos como partidos políticos busquen crear alianzas que les permitan recuperarse rápidamente,

\footnotetext{
${ }^{3}$ Michels, Robert. Los partidos políticos. Buenos Aires: Amorrortu editores, 1996
} 
tanto económica como políticamente, en caso de derrota, o de corresponder al apoyo que se les brindó, en caso de salir victoriosos.

Esta situación, debido a que hace pasar a segundo plano los análisis profundos de la realidad política y social, y las ideologías, da mayor importancia a la imagen que presenten los candidatos durante las elecciones que a los programas de acción que presenten. Dicha imagen, al no poseer más profundidad que la mostrada ante el electorado, puede ser construida con la ayuda de asesores y consultores pagados. De este modo, surgen fenómenos como las campañas negras, y una saturación de publicidad en todos los medios de comunicación posibles, los cuales terminan por dejar en la indiferencia al elector, y lograr el efecto contrario al que buscan, que es precisamente influir en su decisión a la hora de votar.

\section{Procesos electorales}

a. Proceso electoral de 1997

Las elecciones de 1997 tuvieron como característica la verdadera competencia que existió, por vez primera, entre los candidatos de los candidatos de los partidos con mayor militancia, el Partido Revolucionario Institucional (PRI), el Partido Acción Nacional (PAN), y el Partido de la Revolución Democrática (PRD)

Mencionan Poom y Reynoso 4 que en el PRI llegaron a existir hasta doce precandidatos, aunque dos de ellos fueron los más destacados. Uno era Bulmaro Pacheco Moreno, funcionario público con muchos años de militancia, y el otro fue Armando López Nogales, apoyado por el gobernador en turno, Manlio Fabio Beltrones. Después de meses de especulación, fue este último el que llegó a ser candidato por este partido.

\footnotetext{
${ }^{4}$ Poom, Juan, y Victor Reynoso. «Las elecciones de gobernador en Sonora, 1997.» Región y Sociedad 15, № 27,2003, pp. 48-49.
} 
EI PAN enfrentó circunstancias que llegarían serían causas de conflictos, como fue la elección del candidato a la gubernatura del estado Enrique Salgado por parte de la dirigencia nacional, en vez de existir una competencia interna dentro de la militancia estatal.

En el PRD cada uno de los precandidatos tuvo posibilidades de ganar la candidatura debido a la aceptación de que eran objeto entre la militancia del partido, de los cuales resultó victorioso Jesús Zambrano Grijalva, viejo militante de organizaciones de izquierda.

Las campañas iniciaron el 15 de marzo, y duraron 100 días. Las campañas iniciaron con divisiones internas, con largos discursos populistas, y en general, con el sabor de la ventaja del PRI, a causa de los problemas que había enfrentado el PAN con la denominación de su candidato, y el supuesto alejamiento político de Sonora del candidato del PRD. 5

"Armando juntos un mejor futuro con López Nogales" fue el lema del candidato del PRI. A pesar de la ventaja con que iniciaba la campaña tuvo problemas desde el inicio, debido a confusiones y la lucha de intereses entre las distintas corrientes dentro del partido. La imagen que se intentó construir del candidato fue la de un ciudadano que fue aplicado en la escuela, en el trabajo y en su desempeño como funcionario público se intentó presentar como la mejor opción, pero se modificó después esa imagen, ya que el mismo candidato negó haber sido aplicado en la escuela.

En el PAN, Enrique Salgado, pese a hacer su campaña con la mitad de los integrantes de su partido, intentó levantar su campaña. "Por el Sonora que todos queremos ver" fue el slogan del candidato, y gran parte de su proselitismo consistió en un discurso contra el entonces gobernador Manlio Fabio Beltrones Rivera, pero la falta de ofertas concretas, junto con una

5 lbid., p. 57 
imagen poco carismática y su desconocimiento por gran parte del electorado sonorense, hicieron que su campaña no tuviera prácticamente apoyo.

Jesús Zambrano, del PRD, sostuvo el lema "Sonora para todos". La imagen de un político alejado del estado fue siendo sustituía por la de un candidato que buscó proponer, más que criticar, y de intentar construir un partido de izquierda moderna, capaz de hacer acuerdos con empresarios, a diferencia de la primera campaña realizada por el partido en 1991 por Ramón Danzós, en el que se usó una retórica marxista que casi nadie le tomó importancia. Todo esto logró una votación histórica en el estado para su partido, y que no volvería a repetirse.

En los partidos pequeños, la falta de campañas fuertes y eficaces de los partidos grandes los contagió, de modo que no pudieron realizar candidaturas atractivas, lo que provocó que no mostraran el poco o mucho arrastre que hubiesen podido tener.

El 6 de julio de 1997, día de las elecciones, transcurrió con mucha tranquilidad. Armando López Nogales por el PRI se alzó con el triunfo al obtener 304657 votos, muy por encima de los 238500 de Enrique Salgado Bojórquez por el PAN y de los 171300 cobrados por Jesús Zambrano Grijalva del PRD. Pese a que el PRI ganó la gubernatura, tuvo una caída de casi 30 puntos en el porcentaje de votos recibidos respecto a las dos elecciones anteriores. EI PAN obtuvo 31\%, y sorpresivamente el PRD obtuvo más del 22\% de los votos, a diferencia de la elección anterior, donde obtuvo apenas el 3\%. ${ }^{6}$

En ese escenario, el PRI pasó de ser un partido hegemónico a formar parte de un formato tripartidista, ya que, si bien el PRI obtuvo una amplia ventaja sobre los demás partidos en la elección gubernamental, no fue así en 
cuanto a las municipales y a las elecciones para elegir diputados y senadores, ya que en las municipales la oposición ganó 27 de los 73 municipios de Sonora, y el PRI perdió en todas las ciudades importantes, con excepción de Nogales, y en cuanto a las diputaciones locales, el PRI tendría el $42.4 \%$, el PAN tendría el $27.3 \%$ y el PRD el $24.3 \%$, como un reflejo de lo sucedido en el Poder Legislativo Federal. ${ }^{7}$

Según Poom: "La entidad sufrió retardos en la aprobación de reglas que permitieran avanzar de la cultura del fraude a la de la organización electoral con ciudadanos; incentivar la participación social; crear mecanismos para permitir que, en los distritos y municipios, tradicionalmente ganados por el PRI, hubiera alternancia electoral, aunque hoy para ganar las elecciones pesan campañas electorales estratégicas basadas en la mercadotecnia política." 8

b. Proceso electoral de 2003

Las elecciones de 2003, según Poom $^{9}$ y Covarrubias ${ }^{10}$ estuvieron caracterizadas desde el principio por varios motivos: la forma complicada en que se definieron las candidaturas de los partidos políticos principales; sólo el $53 \%$ eligió gobernador; fue la elección más cerrada en la historia de Sonora, ya que el margen de victoria fue de menos de un punto porcentual, y marcó un retroceso al avance político que había mostrado el PRD, y que lo ha seguido en las elecciones subsecuentes. Esto ha tenido como consecuencia que a partir de esta elección se haya creado una suerte de bipartidismo entre elPRI y el PAN en el estado, y que la competitividad se establecería en las elecciones para gobernador.

\footnotetext{
7 Poom, Juan, y Reynoso, Víctor, op.cit., pp.63-65

8 Poom, Juan. «Las elecciones locales en Sonora, 1997-2006. Los alcances de la competitividad electoral.» Región y sociedad Número19,2007, p. 9

9 lbid., pp. 10-11

${ }^{10}$ Covarrubias, Alejandro, op.cit., p.61
} 
Todo comenzó con la irrupción en escena de los grupos políticos de mayor importancia en Sonora a principios del siglo XXI: el "Yaqui Power" y el "centro panista", ambos teniendo como cabezas visibles a Eduardo Bours Castelo del PRI y a Ramón Corral Ávila del PAN, que fueron coordinadores financieros en las campañas presidenciales del 2000 de sus respectivos partidos. ${ }^{11}$

Eduardo Bours poseía una extensa trayectoria en los ámbitos empresariales nacionales, como ser representante agropecuario en las negociaciones del Tratado de Libre Comercio, y dirigir el Consejo Coordinador Empresarial, sin contar los estrechos lazos con el presidente Zedillo y su grupo político. Para llegar a ser candidato del PRI a la gubernatura, tuvo que enfrentarse al candidato oficial, Alfonso Molina Ruibal, lo que dividió a su partido.

Ramón Corral, para ser elegido candidato del PAN tuvo como contrincante a Francisco Búrquez, apoyado por Manuel Espino, Secretario General del PAN, y apoyado por la vieja guardia del PAN en Sonora, lo cual provocó una fractura en el partido, al igual que con el PRI de Bours.

Ya en campaña, rápidamente los candidatos del PRI y del PAN encabezaron la contienda, haciendo de las demás candidaturas meros espectadores. Con anterioridad, Bours y Corral ya se habían enfrentado por una senaduría de mayoría, ganando Ramón Corral, aunque a Bours y al PRI les alcanzó la votación para llegar al Senado como segunda mayoría. Ambos candidatos construyeron estructuras paralelas a las de sus partidos para sus campañas, similar a lo acontecido durante las elecciones presidenciales del 2000, ya que Corral creó la asociación "Amigos de Corral”, dirigida por Padrés 
Elías, y Bours creó la Fundación "Yo con Bours", transformada después en "Yo con Sonora".

Para Poom: "Los ingredientes principales de este período electoral fueron campañas tensas, inequidad en los gastos y cobertura de medios, poca definición de las agencias encuestadoras para acertar estadísticamente en el candidato vencedor y mucho interés nacional y local." 12

Durante las elecciones se comenzó a ver por primera vez de modo tangible el fenómeno de las "Campañas sucias", tejidas por expertos del marketing político. Corral fue asesorado por Santiago Pando, que anteriormente fue asesor de Fox en el 2000. Bours, por su parte, contrató a Don Walter, que anteriormente había aconsejado a diversos políticos a nivel internacional. A Ramón Corral se le imputó de haber estado al tanto de lo ocurrido dentro de "Amigos de Fox", y de que Beltrones estaba detrás de su campaña, con el fin de desprestigiar a Bours, con quien éste había tenido diversos roces. Éste, por su parte, acusó a Eduardo Bours de haber participado en el FOBAPROA y el Pemex-Gate.

En la elección por la gubernatura del 6 de julio de 2003, el PAN, con 364544 votos estuvo cerca de triunfar sobre el PRI, que alcanzó 372472 votos. ${ }^{13}$ Debido a lo reñido del resultado final, se tuvo que definir en el Tribunal Electoral del Poder Judicial de la Federación, el cual declaró ganador a Eduardo Bours Castelo, el 11 de septiembre de 2003, apenas dos días antes de la toma de posesión de éste.

c. Proceso electoral de 2009

\footnotetext{
${ }^{12}$ Poom, Juan, op.cit. p.10

${ }^{13}$ Idem
} 
Según Peñúñuri ${ }^{14}$ el proceso electoral de 2009 confirmó la tendencia creciente a simplificar el discurso político de los candidatos con el propósito de adaptarse a los requerimientos que exige el mercado de los slogans y la carencia de ideas, lo cual contribuyó al aumento del voto nulo y la abstención en el día de los comicios.

Menciona dicho autor que los medios impresos fueron uno de los grandes vencedores tras los cambios realizados a la legislación y la prohibición expresa de contratar publicidad en radio y televisión. Pero no fueron los únicos, ya que la industria de la impresión, las cadenas de proyección cinematográficas, los portales de internet, entre otros, se vieron beneficiados de que el dinero de las campañas pudiera circular más allá de los medios tradicionales, aunque no en un contexto favorable para la libre expresión de las ideas. ${ }^{15}$

En el proceso de elección de candidato del PRI, Ernesto Gándara, quien acababa de renunciar a su puesto de Presidente municipal de Hermosillo para contender por la gubernatura, se enfrentó a Alfonso Elías Serrano. Bours, que tenía en Elías Serrano una suerte de proyecto transexenal, hizo todo por disuadir a Gándara. Al no lograrlo, instrumentó una suerte de elección primaria, en la que Elías derrotó por amplio margen a Gándara (211 000 votos contra 103 000), elección que fue denunciada por estar supuestamente plagada de irregularidades. ${ }^{16}$

Por parte del PAN, Guillermo Padrés Elías ganó con relativa facilidad la contienda interna de su partido a la gubernatura. Padrés Elías era un senador con licencia que había pasado por diversos puestos de elección popular,

${ }_{14}$ Peñúñuri, Amílcar. «Medios de comunicación y elección a gobernador en Sonora 2009: entre la tentación del retorno al pasado y el difícil equilibrio.» Estudios sobre Sonora 2009, 2009, p. 153

${ }^{15}$ Peñúñuri, Amílcar, op.cit., p.154

${ }^{16}$ Covarrubias, Alejandro. op.cit., pp.68-69 
ganado de manera sorpresiva, como en el año 2000, que ganó un puesto de Diputado Federal al haberse enfrentado a una persona de peso en el PRI, Roberto Sánchez Cerezo por el II Distrito Electoral Federal de Sonora.

En cuanto a los demás candidatos, Petra Santos del PRD, Miguel Ángel Haro del PT, y Francisco Casanova Hernández del Partido Social Demócrata (PSD), sus campañas apenas tuvieron repercusión en el electorado sonorense.

Como ocurrió seis años antes, fueron el PRI y el PAN los que acapararon la atención y el apoyo por parte de la ciudadanía sonorense, y de nuevo, como en la elección pasada, los candidatos de ambos partidos ya se habían enfrentado para un puesto de Senador; en este caso Guillermo Padrés derrotó a Alfonso Elías Serrano, quien además era su primo.

Al igual que en las elecciones de 2003, la disputa principal entre el candidato del PAN y el del PRI Alianza no tuvo lugar ni en el terreno programático ni en el ideológico. La disputa se desplazó a los "war rooms" focalizados en el marketing, en las razones de las encuestas día a día, y en las reacciones de las audiencias a una u otra táctica de los "creadores de imagen".

La campaña de Elías Serrano estuvo basada en promover los valores de la cultura sonorense, los valores familiares, en la honra al código vaquero y en la excusa de su carencia de ideas o discursiva, por el hecho de que los sonorenses son gente de acciones, de resultados y donde las palabras no son lo meramente principal para un gobernante. En pocas palabras, en torno a llevar a Sonora "Al Siguiente Nivel", con base en un Sonora "vaquero, "francote", y "echado pa' delante". ${ }^{17}$

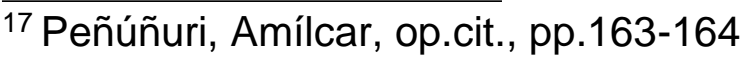


Padrés centró su campaña en decir que Sonora y los sonorenses debían ser los "número uno", por lo que se necesitaba un Sonora Fuerte, un "Nuevo Sonora", el cual fue usado como un lema sencillo, y a su lado la imagen del candidato levantando el dedo índice. Dicha imagen proliferó en miles de pendones por todo el estado. Otra de las propuestas fue la de otorgar uniformes gratuitos y eliminar las cuotas escolares, construir una nueva universidad, mejorar la infraestructura urbana, generar más empleos, apoyar las microempresas y microcréditos, etc. ${ }^{18}$

Del lado de Elías Serrano, estuvieron de asesores un grupo profesional diverso a cargo de Juan Carlos "el Chino" Lam, operador político del gabinete del gobernador. Del lado de Padrés estuvo el mismo publicista que años antes estuvo del lado de Eduardo Bours, Don Walter. Además, en esta campaña fue característico el uso de dos recursos novedosos: el Internet y los jóvenes. Esta fue la primera campaña en que se comenzaron a utilizar de modo más amplio las redes sociales, en especial YouTube, el cual sirvió como punta de lanza para las campañas sucias, así como el uso de un ejército masivo de jóvenes, conocido como "promotores del voto", los cuales realizaban encuestas, levantaban información de los electores, participaban en mítines, etc.

La guerra sucia, al igual que en las elecciones pasadas, se llevó la mayor parte de las energías, spots, anuncios y dinero de los contendientes. Para Elías Serrano se documentaba cómo fluían los recursos de gobierno a través de los exfuncionarios que dirigían su campaña. En cuanto a Padrés, se acusaba a éste de que el ex-gobernador Beltrones estaba detrás de su campaña.

Conforme pasaba la elección, Alfonso Elías Serrano fue perdiendo fuerza en su candidatura, debido a varios factores. En primer lugar, no era un buen candidato, por diversas características como sus problemas durante sus

${ }^{18}$ Ibid., pp.160,162 
mítines (en uno de ellos se equivocó varias veces al momento de nombrar una localidad, siendo el video donde se muestra esto difundido en Internet), y el haber perdido una elección ante Guillermo Padrés. Además, el hecho de que diversos personajes importantes dentro de su partido, los cuales no estaban de acuerdo con la gubernatura de Bours, como su propio hermano Ricardo Bours, y Ernesto Gándara, que incluso operó políticamente en favor de su primo Javier Gándara, del PAN, para la alcaldía de Hermosillo, restaron fuerza a su campaña. ${ }^{19}$

Un último suceso afectaría de tal manera al candidato del PRI que hasta el mismo gobernador en turno mencionó que el modo en que trataron el evento los medios de comunicación fue la causa principal de la derrota de su partido en estas elecciones. ${ }^{20}$ El día 5 de junio, y a unas horas de registrarse un debate entre los candidatos a la gubernatura, se incendió la guardería $A B C$, en Hermosillo, causando la muerte de 49 niños y quemaduras en más de 70 niños.

La tragedia de la Guardería ABC afectó al candidato del PRI, en virtud del manejo que se tuvo del tema por parte de las autoridades estatales, principalmente del gobernador Eduardo Bours, que mostró una actitud en ciertos puntos insensible. Todo ello fue aprovechado por el equipo de Padrés, el cual bajó la intensidad de sus ataques a Elías Serrano, dejando que las implicaciones por la tragedia de la Guardería ABC nublaran su imagen.

Con un $48 \%$ de los votos ganó Guillermo Padrés contra un $44 \%$ de la votación para Alfonso Elías, su competidor en las elecciones de 2006 para Senador por Sonora. Petra Santos, del PRD no alcanzó ni el 4\% de la

${ }^{19}$ Covarrubias, Alejandro. op.cit., p. 69

${ }^{20}$ Peñúñuri, Amílcar, op.cit., pp.156 
Las elecciones para Gobernador en Sonora durante la alternancia política (19972015)

Año 11, Número 20 enero-junio 2019

Francisco Alán, Collins

votación. ${ }^{21}$ De este modo, Guillermo Padrés Elías se convirtió en el primer Gobernador de Sonora proveniente del PAN, y por lo tanto, de la oposición.

\section{d. Proceso electoral de 2015}

Este proceso electoral, en sus características generales, repitió los mismos patrones de los anteriores procesos, ya que se observaron situaciones como la lucha entre precandidatos dentro de los partidos, la intensidad de las campañas negras, y el hecho de ser los candidatos del PAN y del PRI los que realmente se disputaron la gubernatura.

El calendario electoral aprobado por el Instituto Estatal Electoral y de Participación Ciudadana (IEEPC) estableció que el período de campaña electoral de los precandidatos se realizaría entre el 07 de enero al 15 de febrero de 2015, el registro de los candidatos sería del 16 de febrero al 02 de marzo; la campaña propiamente se llevaría a cabo entre el 6 de marzo y el 3 de junio; la veda reglamentaria del 4 al 6 de junio, y la votación el 7 de junio..22

Los principales partidos políticos, el PRI y el PAN, decidieron seleccionar a sus candidatos al gobierno de la entidad vía acuerdos de las dirigencias nacionales, usando la participación de la militancia solamente como una vía de legitimación. ${ }^{23}$

En cuanto al proceso de elección del candidato del PAN, se eligió a Javier Gándara Magaña el domingo 15 de febrero con la participación de casi diecisiete mil militantes, y registró su candidatura el 2 de marzo ante el IEEPC. Cabe destacar que Gándara había iniciado la promoción de su imagen desde

${ }^{21}$ Covarrubias, Alejandro. op.cit., p. 68

22 IEE Sonora. Calendario electoral 2014-2015. 2015. http://www.ieesonora.org.mx/estadistica/2015/informacion/calendario_elector al_2014_2015.pdf (último acceso: 30 de marzo de 2016).

${ }^{23}$ Poom, Juan. "La competencia electoral en Sonora 2015: La disputa de la gubernatura.» 3er. Congreso Internacional de Ciencia Política. Guadalajara: Universidad de Guadalajara, 2015, p.2 
meses atrás, con lo que sus propuestas permearon en la población, haciendo la precandidatura de su contrincante, Francisco García Gámez una suerte de mero trámite.

En el caso del PRI, existieron cuatro precandidatos, los cuales fueron Ernesto Gándara Camou, Claudia Pavlovich Arellano, Antonio Astiazarán Gutiérrez, y Jesús Alberto Cano Vélez, aunque la contienda verdadera se dio entre los dos primeros. Ernesto Gándara tenía como antecedentes el haber sido presidente municipal de Hermosillo de 2006 a 2009, haber sido precandidato a la gubernatura en las elecciones pasadas, y ganar la fórmula para el Senado en 2012. Claudia Pavlovich, hija de la primera senadora y ex alcaldesa de Hermosillo, Alicia Arellano Tapia, había sido regidora, diputada local y senadora por la Fórmula que incluía a Ernesto Gándara en 2012.

Para la elección del candidato, el Comité Ejecutivo Nacional del PRI convocó a un registro de precandidaturas del 16 al 31 de enero de 2015, siendo Claudia Pavlovich elegida el día 27 de enero. Se rumoró que se repitió la misma historia de 2009, cuando Ernesto Gándara, por diversos conflictos tenidos con la jerarquía de su partido, no fue seleccionado para contender como candidato para gobernador. Sin embargo, el día 12 de febrero, en los principales diarios de Sonora (El Imparcial, Expreso, Tribuna) Gándara publicó una carta destinada a la militancia priista, donde rechazaba separarse del partido, para seguir buscando la transformación del mismo. Así el estado de las cosas, el 28 de febrero se inscribió a Claudia Pavlovich como candidata, en una alianza con el Partido Verde Ecologista de México (PVEM) y el Partido Nueva Alianza (PANAL), con el lema "Por un gobierno honesto y eficaz".

En cuanto a los otros partidos, la selección de candidatos pasó con diversas circunstancias. En el caso del PRD, el candidato seleccionado fue Carlos Navarro López, maestro de la Universidad de Sonora y diputado local, después de meses de conflictos internos, que llevó a la salida de 
personalidades como Ana Gabriela Guevara, que había sido considerada para lanzarse a la candidatura, plantearse una coalición con el PAN, partido bastante distinto ideológicamente y la destitución de José Guadalupe Curiel de la dirigencia estatal a consecuencia de esta postura.

En el caso de Movimiento de Regeneración Nacional (MORENA), fue elegido Javier Lamarque Cano, ex militante perredista y alcalde de Ciudad Obregón, debido a su trabajo en el proyecto de Andrés Manuel López Obrador. En cuanto a los demás partidos políticos, las nominaciones de Manuel de Jesús Baldenebro Arredondo, ex priista y ex alcalde de San Luis Río Colorado (Partido Encuentro Social), Jaime Moreno Berry (Partido del Trabajo), y Antonio Pérez Yescas (Partido Humanista), transcurrió sin más complicaciones más que la confirmación de las candidaturas de los dos últimos se dio días después del inicio de las campañas.

Las campañas iniciaron el primer minuto del 6 de marzo de 2015. Este proceso electoral tuvo como una característica la prohibición en las principales ciudades del uso de pendones en las calles, con el fin de evitar la contaminación visual ${ }^{24}$, lo cual no evitó que se siguieran usando los grandes espectaculares, así como los anuncios en la prensa escrita, radio y televisión. Se destacó además el uso intensivo del internet, las redes sociales como Facebook y Twitter, aunque sólo Javier Gándara y Claudia Pavlovich tenían direcciones web.

Desde el principio de las campañas, los candidatos del PAN y del PRI, al igual que en las 2 elecciones pasadas, encabezaron la contienda. Otra característica es que, casi desde el inicio, surgieron las campañas negras. Como señala Poom: "Lo que en principio inició con ideas asociadas a temas de infraestructura, innovación en la gestión pública [...] que los candidatos empezaban a posicionar en sus agendas de campaña, empezó a ser rebasado

$$
{ }^{24} \text { lbid., p.10 }
$$


por la nube mediática y certera de la descalificación personal, la denigración, la pobreza de argumentos sobre los temas que los ciudadanos esperaban escuchar." 25

El PT y el PRI fueron los primeros en iniciar las denuncias, publicando en diversos medios irregularidades por parte del ex alcalde de Hermosillo durante su gestión, y dando a conocer incluso un reportaje del Wall Street Journal, en el cual se publicó "se investigan presuntas irregularidades del gobernador Guillermo Padrés Elías". Cabe mencionar que el día 17 de marzo, la candidata Claudia Pavlovich, junto con diversas personalidades de su partido, asistieron a la Plaza Zaragoza, ubicada frente al Palacio de Gobierno, llevando documentación que comprobaba sus acusaciones. ${ }^{26}$

Conforme pasaron los días, siguieron los ataques del PRI contra el candidato del PAN, en cuestiones de malos manejos administrativos y de ser favorecido por las autoridades electorales. A esto, el candidato del PAN comenzó con acusaciones de clientelismo por parte de la candidata del PRI, mediante grabaciones telefónicas de Pavlovich, además de denuncias por propiedades irregulares e incluso, atacando la imagen de la candidata, por lo cual Claudia Pavlovich convocó a una conferencia de prensa para desmentir estas acusaciones. ${ }^{27}$

Como dato por demás curioso, en el caso del Partido Humanista, además de su nula actividad proselitista, Antonio Pérez Yescas renunció a la

\footnotetext{
25 Ibid., p.11

${ }^{26}$ Revista Expansión,Sección Nacional, El gobierno federal investiga al gobernador de Sonora, según WSJ," 2015[en línea]disponible en: https://expansion.mx/nacional/2015/03/05/el-gobierno-federal-investiga-algobernador-de-sonora-segun-wsj; consultado el 19 de Enero de 2019 ${ }^{27}$ Revista Milenio,Sección Estados,Ataja Claudia Pavlovich críticas de su contrincante de PAN, 2015[en línea]disponible en: https://www.milenio.com/estados/ataja-claudia-pavlovich-criticascontrincante-pan; consultado el 19 de Enero de 2019
} 
candidatura por este partido, por lo que se le sustituyó a media campaña por Héctor Castro Leal, el cual terminó por apoyar a la candidatura de Javier Gándara.

Además de las campañas, se realizaron dos debates organizados por el IEEPC los días 21 de abril y 19 de mayo. El primero se llevó a cabo en el Salón de Convenciones del Hotel Royal Palace, en la ciudad de Hermosillo, y el segundo en la Sala Club ITSON, en Ciudad Obregón. Estos debates se caracterizaron, además por ser más una exposición de ideas que una discusión de las mismas, por ser una especie de continuación de las denominadas campañas negras, donde las denuncias, las acusaciones, prevalecieron por sobre las propuestas.

Conforme se acercaba el 7 de junio, día de la votación, la campaña negra subió de nivel. Uno de los incidentes más recordados fue la cuelga de 2 mantas en el bulevar Luis Encinas, en la ciudad de Hermosillo, las cuales tenían mensajes denigrantes hacia la mujer. El primero de ellos tenía la leyenda: "La panocha en las coyotas, ¡No en Palacio!", y la segunda rezaba: "Las mujeres como los fusiles: cargadas y en el rincón." ${ }^{28}$ El PAN inmediatamente se deslindó de tales mantas.

El domingo 7 de junio se realizaron las elecciones para elegir al gobernador que regiría en el período 2015-2021, además de los 72 ayuntamientos, y las diputaciones tanto locales como federales. Con excepción de algunos incidentes aislados, la jornada electoral se llevó a cabo en un ambiente de tranquilidad.

${ }^{28}$ Periódico Excelsior, En Sonora, comparan a mujeres con escopetas: “cargadas y en el rincón" 2015 [en línea]disponible en: https://www.excelsior.com.mx/nacional/2015/04/30/1021739 consultado el 30 de marzo de 2019 
Fue en la noche cuando surgieron complicaciones, debido a que se esperaba que a las 22:00 de ese mismo día se anunciara por el IEEPC un conteo rápido, el PREP (Programa de Resultados Preliminares), pero debido a la lentitud con la que fueron llegando los paquetes con las boletas electorales y con la que se procesaron, no se pudieron brindar resultados fiables sino hasta las primeras horas del día siguiente, los cuales comenzaban a favorecer a Claudia Pavlovich. Mientras tanto, Javier Gándara anunció en una conferencia de prensa que los primeros resultados del conteo le daban una ventaja de $1.8 \%$, pero que esperaría a que el conteo concluyera para alzarse con la victoria.

Fue hasta el día 13 de junio cuando se confirmó que la candidata del Partido Revolucionario Institucional había ganado las elecciones, con 440507 votos, que representaban el $48.57 \%$, contra 415745 votos de Javier Gándara, que representaban el $41.61 \%$ de la votación total. En cuanto a los otros partidos, tanto el PRD como MORENA obtuvieron apenas la votación suficiente para mantener su registro, mientras que el resto de los partidos perdieron su registro al no alcanzar suficientes votos. ${ }^{29}$

EI PAN, en los días siguientes de la elección, movilizó a ciudadanos frente a las oficinas del IEEPC para que se hiciera el reconteo de los paquetes electorales. Se alegaba que, debido a indicios de irregularidades, la votación había sido favorecida hacia Claudia Pavlovich. Pero el día 13 de junio, mismo día en que se declaró la victoria de Pavlovich, el presidente del Comité Directivo Estatal del PAN, Juan Valencia Durazo, anunció que no se impugnaría la elección. Esto permitió que sin complicaciones se pudiese entregar la constancia de ganadora a Claudia Pavlovich el 15 de junio, y su

${ }^{29}$ IEE Sonora. Resultados del cómputo en elecciones municipales y para gobernador. 2015[en linea]Disponible en:

http://www.ieesonora.org.mx/estadistica/2015/informacion/resultados/ComputoMpal Gob2015.pdf (consultado el 30 de marzo de 2016) 
Las elecciones para Gobernador en Sonora durante la alternancia política (19972015)

Año 11, Número 20 enero-junio 2019

Francisco Alán, Collins

toma de protesta el 13 de septiembre como la primera mandataria mujer en la historia de Sonora.

\section{CONCLUSIONES}

El panorama político en Sonora desde 1997 a la fecha ha visto una transformación en cuanto al papel de los partidos políticos en la vida pública del estado, pasando de un sistema de partido hegemónico a uno bipartidista.

Las elecciones para gobernador de 1997 fue la única ocasión en que las tres principales fuerzas políticas del país, el PRI, el PAN, y el PRD obtuvieron un porcentaje significativo de la votación. Las elecciones de 2003, 2009 y 2015 vieron el desplazamiento del PRD como partido político con apoyo efectivo, pasando a obtener votaciones menores a los diez puntos porcentuales, así como el surgimiento de un bipartidismo representado por el PRI y el PAN, los cuales se han disputado realmente la gubernatura, y contiendas cada vez más cerradas, al punto de llegar a tribunales federales para decidir al ganador, en el caso de la elección de 2003 que se decidió a favor de Eduardo Bours.

Otro aspecto relevante fueron las campañas negras. Éstas han surgido debido a que, con motivo de la apertura democrática tanto a nivel federal como nivel estatal en la década de 1990, la participación de la ciudadana en los procesos electorales ha aumentado, y por consiguiente, la importancia de la opinión pública, por lo que tener una imagen política positiva o negativa llega a ser decisiva a la hora de captar votos. Esto también es causado por elecciones cada vez más cerradas entre los candidatos punteros, que han sido del PAN y el PRI desde 2003 buscándose restar votos, aunque sean mínimos, al otro candidato puntero, en vez de captarlos. Esto provoca que las campañas basadas en ataques lleguen a tener más importancia que las campañas basadas en propuestas de los candidatos. 
Asimismo, siguiendo el patrón presentado en las últimas tres elecciones, se destaca el papel que los medios de comunicación tienen en los procesos electorales, ya que éstos cobran cada vez más importancia, y el hecho de que los partidos políticos tienden a destinar más recursos para formar una imagen carismática de su candidato. Esto por la tendencia del electorado a votar más por el candidato que por el partido, lo que lleva a que sólo los partidos políticos con fuerza en el estado (PRI y PAN) puedan destinar grandes recursos para formar la imagen de su candidato, a diferencia de los partidos más pequeños, que privilegiarán una ideología o mensaje a una imagen.

Como reflexión última, sería interesante analizar si las siguientes elecciones estatales siguen con los mismos patrones, o se presenta un cambio sustancial en la manera en que se llevan a cabo.

\section{Referencias}

COVARRUBIAS, Alejandro. «La alternancia en Sonora. De legacías y divisiones a la cartelización de la política.» En Estudios sobre Sonora 2010, de Eloy Méndez y Alejandro Covarrubias, 49-77. Hermosillo: Mora-Cantúa Editores, 2011.

IEE SONORA. Calendario electoral 2014-2015. 2015.

http://www.ieesonora.org.mx/estadistica/2015/informacion/calendario_ electoral_2014_2015.pdf (último acceso: 30 de marzo de 2016).

- Resultados del cómputo en elecciones municipales y para gobernador. 2015.

http://www.ieesonora.org.mx/estadistica/2015/informacion/resultados/ ComputoMpalGob2015.pdf (último acceso: 30 de marzo de 2016).

MICHELS, Robert. Los partidos políticos. Buenos Aires: Amorrortu editores, 1996. 
PEÑÚÑURI, Amílcar. «Medios de comunicación y elección a gobernador en Sonora 2009: entre la tentación del retorno al pasado y el difícil equilibrio.» Estudios sobre Sonora 2009, 2009: 153-168.

PERIÓDICO EXCELSIOR En Sonora, comparan a mujeres con escopetas: "cargadas y en el rincón," 2015[en línea]disponible en: https://www.excelsior.com.mx/nacional/2015/04/30/1021739 consultado el 30 de noviembre de 2018

POOM, Juan. «La competencia electoral en Sonora 2015: La disputa de la gubernatura.» 3er. Congreso Internacional de Ciencia Política. Guadalajara: Universidad de Guadalajara, 2015 «Las elecciones locales en Sonora, 1997-2006. Los alcances de la competitividad electoral.» Región y Sociedad, no.19, 2007

POOM, Juan, y Víctor Reynoso. «Las elecciones de gobernador en Sonora, 1997.» Región y Sociedad, № 27,2003

REVISTA EXPANSIÓN, Sección Nacional, El gobierno federal investiga al gobernador de Sonora, según WSJ," 2015[en línea]disponible en: https://expansion.mx/nacional/2015/03/05/el-gobierno-federalinvestiga-al-gobernador-de-sonora-segun-wsj (consultado el 19 de noviembre de 2018)

REVISTA MILENIO, Sección Estados, Ataja Claudia Pavlovich críticas de su contrincante de PAN, 2015[en línea]disponible en:

https://www.milenio.com/estados/ataja-claudia-pavlovich-criticascontrincante-pan (consultado el 19 de noviembre de 2018) 